Contact: Dr Xiu Fen Chen.

E-mail: xiufen.chen@mohh.com.sg

1. McMenamin ME, Fletcher CDM. Reactive angioendotheliomatosis a study of 15 cases demonstrating a wide clinicopathologic spectrum. Am J Surg Pathol 2002; 26: 13

2. Rongioletti F, Rebora A. Cutaneous reactive angiomatoses: patterns and classification of reactive vascular proliferation. J Am Acad Dermatol 2003; 49: 887-96.

3. Dispenzieri A, Kyle RA, Lacy MQ, et al. POEMS syndrome: definitions and long-term outcome. Blood 2003; 101: 2496-506

4. Dispenzieri A. How I treat POEMS syndrome. Blood 2012; 119: 5650-8.

5. Krell JM, Sanchez RL, Solomon AR. Diffuse dermal angiomatosis: variant of reactive cutaneous angioendotheliomatosis. J Cutan Pathol 1994; 21: 363-70

6. Suurmeijer AJH, Fletcher CDM. Papillary haemangioma. A distinctive cutaneous haemangioma of the head and neck area containing eosinophilic hyaline globules. Histopathology 2007; 51: 638-48.

7. Maloney N, Miller P, Linos K. Papillary hemangioma: an underrecognized entity not to be confused with glomeruloid hemangioma. Am J Dermatopathol 2020; 42: 211-4.

8. Chan JKC, Fletcher CDM, Hicklin GA, et al. Glomeruloid hemangioma a distinctive cutaneous lesion of multicentric Castleman's disease associated with POEMS syndrome. Am J Surg Pathol 1990; 14: 1036-46.

9. Singer C, Mallon D, Auguston B, et al. Reactive angioendotheliomatosis presenting as livedo racemosa secondary to propylthiouracil. Pathology 2020; 52: 494-6.

10. Creamer D, Black MM, Calonje E. Reactive angioendotheliomatosis in association with the antiphospholipid syndrome. J Am Acad Dermato 2000; 42: 903-6.

11. Marinho FS, Pirmez R, Nogueira R, et al. Cutaneous manifestations in POEMS syndrome: case report and review. Case Rep Dermatol 2015; 7 $61-9$

12. Tsai CY, Lai CH, Chan HL, et al. Glomeruloid hemangioma-a specific cutaneous marker of POEMS syndrome. Int J Dermatol 2001; 40: 403-6.

DOI: https://doi.org/10.1016/j.pathol.2020.07.016

\section{Malignant gastrointestinal neuroectodermal tumour in soft tissue}

Sir,

Malignant gastrointestinal neuroectodermal tumour (GNET) is an uncommon and aggressive primitive mesenchymal neoplasm of the digestive tract, predominantly occurring in young to middle aged adults (median age 35 years), with no obvious gender predominance. Malignant GNET is also known as clear cell sarcoma-like tumour of the gastrointestinal tract, because it shares with clear cell sarcoma of soft tissues (CCS-STs) a similar morphological appearance and a common genetic characteristic of EWSR1-ATF1/CREB1 fusion genes resulting from $\mathrm{t}(12 ; 22)(\mathrm{q} 13 ; \mathrm{q} 12)$ or $\mathrm{t}(2 ; 22)$ (q34; q12). ${ }^{1}$ The recent reports have presented several novel confirmed or suspected cases, respectively located in palate, tongue $^{3}$ and bronchus, ${ }^{4}$ but malignant GNET with extragastrointestinal origin is actually extremely rare. Herein, we present an unusual case of malignant GNET arising in the deep soft tissue of the thigh, a hitherto unreported occurrence site for the tumour. We briefly discuss the clinicopathological features of malignant GNET.

A 47-year-old female presented with a subcutaneous mass in her right thigh for 10 years without local swelling, tenderness, skin ulceration, stiffness or difficult movement. The mass had increased slightly in size over several months. Imaging included ultrasound scan, magnetic resonance imaging and digital radiography and showed a $6 \mathrm{~cm}$ well defined soft tissue mass, located in the intermuscular space between the adductor magnus and sartorius muscle on the right. However, anterio-posterior and lateral radiograph of the right femur indicated no skeletal abnormality. Additionally, there was no evidence of regional lymph node metastasis and distant metastasis. Her physical examination and laboratory findings were unremarkable except for serology analysis which showed that the patient was positive for the hepatitis B surface antigen (HBsAg) and hepatitis B core antibody (HBcAb). Abdominal ultrasound and computed tomography (CT) scan of the abdomen and pelvis revealed no mass in the gastrointestinal tract.

Surgical excision of the mass was performed. Macroscopic examination revealed a greyish-red lobulated mass measuring $6 \times 5 \times 4.5 \mathrm{~cm}$; the mass was slightly firm, smooth surfaced, and not obviously adherent to surrounding tissue. No haemorrhage or necrosis was observed on multiple cut sections. Microscopically, the tumour was well circumscribed with a multinodular appearance separated by fibrous connective tissue (Fig. 1A). The tumour nodule was composed of a proliferation of uniform spindle cells arranged in short fascicles (Fig. 1B). The tumour cells displayed abundant predominantly clear cytoplasm, fine nuclear chromatin, and indistinct nucleoli (Fig. 1C). Mitoses were very rare. There was focal cystic change (Fig. 1D). Characteristic osteoclastlike giant cell was not identified. Immunohistochemistry staining showed that the tumour cells were strongly and diffusely positive for S-100 protein (Fig. 1E) and SOX10 (Fig. 1F), weakly positive for CD99, focally and weakly positive for CD56 and EMA, while negative for HMB45, Melan A, synaptophysin, Chromogranin A, CD117, CD34, DOG-1, CK, SMA, desmin, myogenin, and myoD1. Ki-67 proliferation index was about 5\%. Fluorescence in situ hybridisation (FISH) analysis demonstrated the presence of EWSR1 rearrangement and EWSR1-ATF1 fusion (Fig. 2), while the other common gene fusions involving EWSR1, including EWSR1-FLI1, EWSR1-CREB1 and EWSR1-WT1, were all absent.

Given that the case occurred in soft tissue of a lower extremity and was characterised by spindle cell appearance, our differential diagnoses mainly included CCS-STs, monophasic synovial sarcoma and malignant peripheral nerve sheath tumour (MPNST). Malignant GNET shares overlap with CCS-STs in morphological appearance and genetic abnormalities. Nevertheless, CCS-STs harbours melanocytic differentiation and is variably positive for HMB45, Melan-A or MiTF, ${ }^{5}$ while all reported cases of malignant GNET, including our case, are unexceptionally lacking expression of melanocytic markers. Monophasic SS histologically exhibits fascicles of monomorphic spindled cells without an epithelial component, which should be differentiated from our case. Although S-100 protein has been observed in $10-40 \%$ of SS cases, its expression is often focal ${ }^{6-8}$ instead of the diffuse and strong staining pattern in malignant GNET. ${ }^{1}$ The detection of SYT gene rearrangement resulting from specific chromosomal translocation $\mathrm{t}(\mathrm{X} ; 18)(\mathrm{p} 11.2 ; \mathrm{q} 11.2)$ can aid in confirming the diagnosis of a synovial sarcoma. MPNST is characterised by a proliferation of spindle cells with alternating hypercellular and hypocellular areas, and is ordinarily positive for the neural markers including S-100 protein, Leu-7, PGP9.5 and myelin 

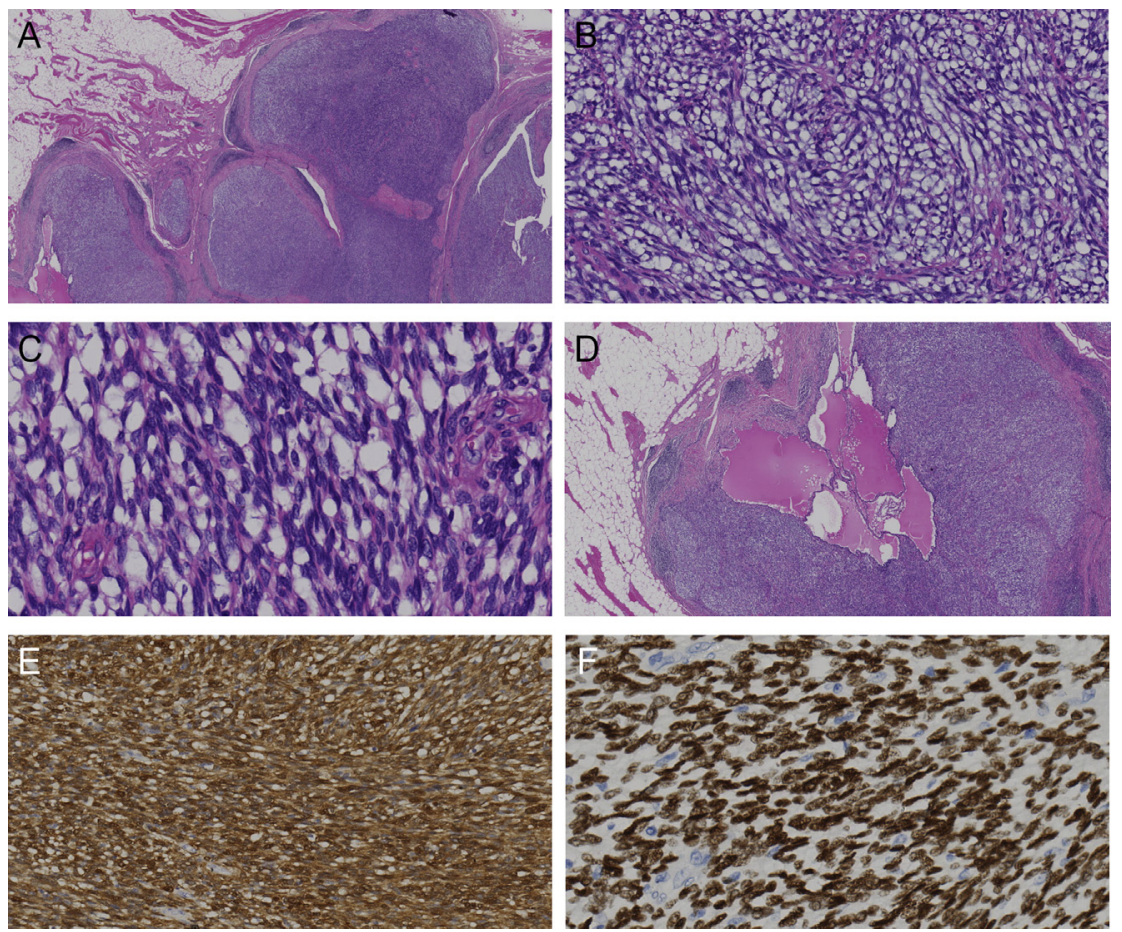

Fig. 1 (A) Multinodular structure separated by fibrous connective tissue (H\&E). (B) Tumour cells were arranged in a fascicular pattern (H\&E), and (C) presented a short fusiform shape with mostly clear cytoplasm, fine nuclear chromatin and inconspicuous nucleoli (H\&E). (D) Focal cystic structure was present (H\&E). (E) Strong diffuse S-100 protein staining (IHC). (F) Strong diffuse SOX10 staining (IHC).

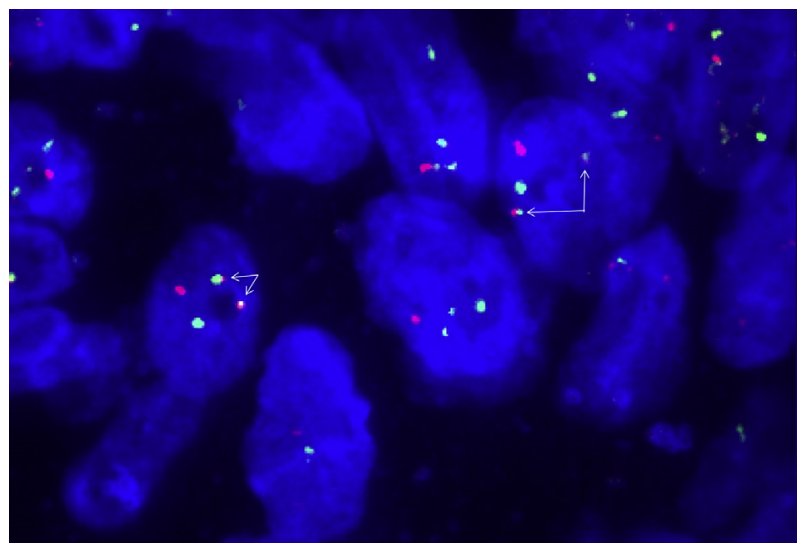

Fig. 2 Representative image of EWSRI-ATF1 fusion by FISH analysis. The tumour cells showed two fusion signals (indicated by the white arrows), and a separate red and green signal (1R1G2F).

basic protein. However, S-100 protein is also frequently patchily and moderately positive in MPNST. ${ }^{6,8}$ Additional important diagnostic clues include that a MPNST mass may be closely connected to a nerve and typically occurs in a patient with a history of neurofibromatosis, so these could be easily excluded. Moreover, the patient did not exhibit digestive system symptoms such as abdominal pain, abdominal distension, vomiting, melena or intestinal obstruction. Physical examination of the abdomen also revealed unremarkable findings. Particularly, the patient had no evidence of gastrointestinal mass based on imaging findings including ultrasound and CT. Therefore, the possibility of tumour metastasis from the gastrointestinal tract could be excluded.
Finally, based on the clinicopathological features, immunophenotypes and molecular genetic findings, the tumour was diagnosed as malignant GNET in soft tissue.

Malignant GNET typically consists of primitive appearing round or polygonal epithelioid cells with a variable amount of lightly eosinophilic or clear cytoplasm, frequently arranged in nested, sheet-like, pseudopapillary, or pseudoalvelar patterns. Occasionally, an uncommon microcystic or rosette-like architecture can be noted. Moreover, a case of oncocytic variant of malignant GNET has been reported. ${ }^{9}$ In a few cases, focal spindle cells components have been observed, ${ }^{1}$ while our case showed purely spindle cell morphology with mostly clear cytoplasm, and lack of epithelioid appearance. The presence of scatter osteoclast-like giant cells is a frequent morphological feature of malignant GNET, but they were not observed in some cases reported, ${ }^{1,9,10}$ just as in our case.

The immunophenotypic profile of the neoplastic cells is characterised by always strong and diffuse staining for S100 protein, positivity for SOX 10 in the majority of reported cases, variable positivity for neuroendocrine markers (NSE, synaptophysin, CD56, and NB84), and invariable negativity for melanocyte specific markers (HMB45, Melan A, tyrosinase, and MiTF). ${ }^{1}$ The aetiology of malignant GNET remains enigmatic. However, based on the accumulating evidence that melanocyte-specific markers are consistently absent while primitive neural markers are often expressed in varying extent, it is assumed to possibly originate from the gastrointestinal tract autonomic nervous system related primitive cell of neural crest derivation that has lost the potential to differentiate along the melanocytic lineage. ${ }^{1,10}$ Likewise, ultrastructural examination can also demonstrate features of primitive neuroectodermal cells, with no evidence of melanogenesis as well as myoid or any other 
specific differentiation. ${ }^{1,10}$ Therefore in 2012, Stockman et al. first designated the distinct entity as 'malignant gastrointestinal neuroectodermal tumour'.

According to available current data, the biological behaviour of malignant GNET is highly malignant and almost all of the reported cases followed an aggressive clinical course with local recurrence in the short term, and metastasis to lymph nodes or hematogenous spread early or even at initial diagnosis. However, our case presented an indolent clinical course. The patient had evidence of the tumour from the age of 37, which progressed slowly for 10 years without any medical intervention. After excision of the tumour, the patient was alive without evidence of disease for 8 months. Interestingly, Prado et al. have reported one case of indolent GNET of the colon which also had a 10 year long clinical course. ${ }^{11}$ Our case still needs further follow-up observation to evaluate long-term outcome.

Conflicts of interest and sources of funding: The authors state that there are no conflicts of interest to disclose.

\section{Yang Yang ${ }^{1}$, Yanyang Chen ${ }^{1}$, Shaoyu Chen ${ }^{2}$, Anjia Han \\ ${ }^{1}$ Department of Pathology, The First Affiliated Hospital, Sun Yat-Sen University, Guangzhou, China; ${ }^{2}$ Guangzhou LBP Medical Technology Co. Ltd., Guangzhou, China}

Contact Dr Anjia Han.

E-mail: hananjia@mail.sysu.edu.cn

1. Stockman DL, Miettinen M, Suster S, et al. Malignant gastrointestinal neuroectodermal tumor: clinicopathologic, immunohistochemical, ultrastructural, and molecular analysis of 16 cases with a reappraisal of clear cell sarcoma-like tumors of the gastrointestinal tract. Am J Surg Pathol 2012; 36: 857-68

2. Allanson BM, Weber MA, Jackett LA, et al. Oral malignant gastrointestinal neuroectodermal tumour with junctional component mimicking mucosal melanoma. Pathology 2018; 50: 648-53.

3. Breton S, Dubois M, Geay JF, et al. Clear cell sarcoma or gastrointestinal neuroectodermal tumor (GNET) of the tongue? Case report and review of the literature of an extremely rare tumor localization. (French). Ann Pathol 2019; 39: 167-71

4. Zheng Q, Chen H, Li Y. Primary gastrointestinal-type clear cell sarcoma-like tumor of the bronchus: a hitherto unreported bronchial tumor. J Thorac Oncol 2019; 14: e202-5.

5. Hisaoka M, Ishida T, Kuo TT, et al. Clear cell sarcoma of soft tissue: a clinicopathologic, immunohistochemical, and molecular analysis of 33 cases. Am J Surg Pathol 2008; 32: 452-60.

6. Folpe AL, Schmidt RA, Chapman D, Gown AM. Poorly differentiated synovial sarcoma: immunohistochemical distinction from primitive neuroectodermal tumors and high-grade malignant peripheral nerve sheath tumors. Am J Surg Pathol 1998; 22: 673-82.

7. Pelmus M, Guillou L, Hostein I, Sierankowski G, Lussan C, Coindre JM. Monophasic fibrous and poorly differentiated synovial sarcoma: immunohistochemical reassessment of $60 \mathrm{t}(\mathrm{X} ; 18)(\mathrm{SYT}-\mathrm{SSX}$ )positive cases. Am J Surg Pathol 2002; 26: 1434-40.

8. Olsen SH, Thomas DG, Lucas DR. Cluster analysis of immunohistochemical profiles in synovial sarcoma, malignant peripheral nerve sheath tumor, and Ewing sarcoma. Mod Pathol 2006; 19: 659-68.

9. Boland JM, Folpe AL. Oncocytic variant of malignant gastrointestinal neuroectodermal tumor: a potential diagnostic pitfall. Hum Pathol 2016 57: $13-6$.

10. Antonescu CR, Nafa K, Segal NH, Dal Cin P, Ladanyi M. EWS CREB1: a recurrent variant fusion in clear cell sarcoma-association with gastrointestinal location and absence of melanocytic differentiation. Clin Cancer Res 2006; 12: 5356-62.

11. Prado G, Gorcey SA, Szallasi A, et al. Indolent gastrointestinal neuroectodermal tumor (GNET) of the colon: a new entity. J Gastrointest Cancer Stromal Tumor 2017; 2: 2.

DOI: https://doi.org/10.1016/j.pathol.2020.06.021

\section{Parietal cell/oncocytic/ mitochondrion-rich gastric carcinoma: a need for clarity in terminology}

\section{Sir,}

In 1984 Capella et al. introduced three cases of a newly observed variant of gastric adenocarcinoma, which they named parietal cell gastric carcinoma (PCGC). ${ }^{1}$ A small number of case reports followed this first description of the entity until 2002. ${ }^{2}$ PCGCs are primary adenocarcinomas of the stomach that display a glandular or solid pattern of growth occasionally mimicking lymphomas or mesenchymal tumours of smooth muscle origin. ${ }^{2}$ Neoplastic cells are usually polygonal with round nuclei and oncocytic features with abundant eosinophilic, granular cytoplasm reactive with phosphotungstic acid haematoxylin (PTAH) and Luxol fast blue. ${ }^{2-4}$ Commonly, immunohistochemical stains for antimitochondrial antibodies are strongly positive. ${ }^{2,3}$ The ultrastructural features of tumour cells correspond to an 'oncocytic cytoplasm' with abundant mitochondria, tubulovesicles, intercellular lumina filled with undulating microvilli and intracellular canaliculi. ${ }^{2-5}$

In 2002 Takubo et al. presented 10 cases of gastric adenocarcinoma with morphological features similar to those of parietal cell carcinoma, however immunohistochemical staining of the tumours with four different antiparietal cell antibodies (anti- $\mathrm{H}^{+}-\mathrm{K}^{+}$-adenosine triphoshatase antibodies) was negative in all cases. ${ }^{6}$ They claimed that, if the $\mathrm{H}^{+} / \mathrm{K}^{+}$-ATPase proton pump is considered an important property of normal parietal cells, then the 10 carcinomas they encountered could not be said to have shown parietal cell differentiation. Therefore, they used the term oncocytic carcinoma, or carcinoma with oncocytic differentiation, to describe the presented neoplasms. ${ }^{6}$ They also suggested that their cases may represent a different histological subtype from parietal cell carcinoma, however none of the previously reported cases of parietal cell carcinoma had been tested with antiparietal cell antibodies. ${ }^{6}$ Subsequently, in 2005, the same group reported another four cases of the same type of gastric adenocarcinoma and, again, negative staining with antiparietal cell antibodies. ${ }^{7}$ Additionally, they emphasised similarities of these cases with malignant oncocytomas to suggest the term oncocytic carcinomas or carcinomas with oncocytic differentiation.

In 2003 Yang et al. presented the case of a gastric tumour with both parietal cell and incomplete intestinal type differentiation, and found that the tumour cells were strongly and extensively positive for parietal cell biomarkers $\mathrm{H}^{+} / \mathrm{K}^{+}$ATPase and HMFG2 (human milk fat globule-2). ${ }^{5}$ They agreed with the necessity of immunostaining for parietal cell markers to confirm a morphological suspicion of parietal cell differentiation. ${ }^{5}$ HMFG-2/platelet derived growth factor receptor- $\alpha$ (PDGFR $\alpha)$ is currently considered a typical biomarker of parietal cell differentiation.

Following the publication by Takubo in 2002, the terms 'parietal cell' and 'oncocytic gastric carcinoma' have been alternately used in the literature, however it still remains unclear whether the terms represent different entities. ${ }^{2}$ Additionally, it has been suggested recently that there may be a partial overlap of such neoplasms with 'gastric adenocarcinoma of the fundic gland type with predominant parietal cell differentiation'. 